\title{
Comparison of two types of synchronization of external-cavity semiconductor lasers
}

\author{
A. Locquet* \\ Service d'Electromagnétisme et de Télécommunications, Faculté Polytechnique de Mons, 31 Boulevard Dolez, B-700o Mons, Belgium \\ C. Masoller \\ Instituto de Física, Facultad de Ciencias, Universidad de la República, Igua 4225, Montevideo 11400, Uruguay
}

\section{P. Mégret and M. Blondel}

Service d'Electromagnétisme et de Télécommunications, Faculté Polytechnique de Mons, 31 Boulevard Dolez, B-70oo Mons, Belgium

Received July 5, 2001

\begin{abstract}
We study numerically the synchronization of external-cavity semiconductor lasers in a master-slave configuration, based on a Lang-Kobayashi-type model. Depending on the feedback and coupling strengths, the slave laser synchronizes with the injected optical field or with the injected field but lags in time. We show that these two types of synchronization present different robustness with respect to the noise, frequency detuning, and current modulation of the master laser. (C) 2002 Optical Society of America

OCIS codes: $140.5960,140.1540,060.4510$.
\end{abstract}

The synchronization of unidirectionally coupled semiconductor lasers is an issue that has attracted a lot of attention in recent years ${ }^{1-14}$ because of its potential application to encoded optical communications. Locquet et al. ${ }^{15}$ recently showed that, when the lasers are both external-cavity lasers, two different types of synchronization are possible. In the first type, both lasers are subject to the same feedback level, and the slave-laser intensity, $I_{s}(t)$, synchronizes with the intensity injected from the master laser, $I_{m}\left(t-\tau_{c}\right)$, where $\tau_{c}$ is the flight time from the master to the slave laser. In the second type of synchronization, the slave-laser intensity synchronizes with the masterlaser intensity but with a lag time that is the difference between the external-cavity round-trip time, $\tau$, and the flight time from one laser to the other: $I_{s}(t)=I_{m}\left(t-\tau_{c}+\tau\right)$. Ahlers et al. ${ }^{4}$ have shown that this type of synchronization occurs when the feedback level of the master laser, $\gamma_{m}$, is equal to the sum of the feedback level of the slave laser, $\gamma_{s}$, and the optical coupling strength, $\eta$. In this Letter, we study these two types of synchronization and show that they present different sensitivities to noise and frequency detuning and that they differ in the response of the slave laser to current modulation of the master laser.

The rate equations for two single-mode semiconductor lasers with coherent optical feedback and optical injection from one laser to the other are ${ }^{11}$

$$
\begin{aligned}
\dot{E}_{i}= & k_{i}\left(1+i \alpha_{i}\right)\left(G_{i}-1\right) E_{i}(t)+\gamma_{i} E_{i}(t-\tau) \exp \left(-i \omega_{i} \tau\right) \\
& +\eta E_{m}\left(t-\tau_{c}\right) \exp \left[-i\left(\omega_{m} \tau_{c}-\Delta \omega t\right)\right]+\sqrt{D_{i}} \xi_{i}(t),
\end{aligned}
$$

$$
\dot{N}=\left(j_{i}-N_{i}-G_{i}\left|E_{i}\right|^{2}\right) / \tau_{n i} .
$$

Here, the indices $i=m$ and $i=s$ refer to the master and the slave laser, respectively, $E_{i}$ is the slowly varying complex field, and $N_{i}$ is the normalized carrier density. The term $\eta E_{m}\left(t-\tau_{c}\right) \exp \left[-i\left(\omega_{m} \tau_{c}-\Delta \omega t\right)\right]$ in Eq. (1) exists only for the slave laser and accounts for the light injected from the master laser. The parameters are as follows: $k_{i}$ is the cavity losses, $\alpha_{i}$ is the linewidth-enhancement factor, $G_{i}=N_{i} /\left(1+\epsilon_{i}\left|E_{i}\right|^{2}\right)$ is the optical gain (where $\epsilon_{i}$ is the gain saturation coefficient), $\omega_{i}$ is the optical frequency of the solitary laser, and $\Delta \omega=\omega_{m}-\omega_{s}$ is the frequency detuning between the lasers. $\xi_{i}(t)$ are independent complex Gaussian white noises with correlation $\left\langle\xi_{i}(t) \xi_{j}{ }^{*}\left(t^{\prime}\right)\right\rangle=\delta_{i, j} \delta(t-$ $t^{\prime}$ ), and $D_{i}$ is a measure of the noise intensity. $j_{i}$ is the normalized injection current, and $\tau_{n i}$ is the carrier lifetime. The model includes a single reflection in the external cavity and therefore is valid for weak and moderate feedback levels. We assume in the following that the lasers have the same parameters, except for the optical frequencies and the feedback levels.

To characterize the quality of the two types of synchronization, we calculate the correlation coefficient, $C$, between $I_{m}\left(t+\tau_{l}\right)$ and $I_{s}(t)$, where $\tau_{l}=-\tau_{c}$ when $\gamma_{m}=\gamma_{s}$ and $\tau_{l}=\tau-\tau_{c}$ when $\gamma_{m}=\gamma_{s}+\eta$. The parameters used are given in the caption of Fig. 1. In particular, we have chosen a master feedback level $\gamma_{m}=20 \mathrm{~ns}^{-1}$, which leads to a chaotic master-laser intensity.

Figure 2 shows the results when $\gamma_{s}=\gamma_{m}$. The horizontal axis is the frequency detuning between the lasers, the vertical axis is the optical coupling strength, and the gray level represent the value of the correlation coefficient (darker gray represents a large correlation). Figure 2 shows the results for the same parameters as Fig. 1 but when $\gamma_{s}=\gamma_{m}-\eta$. This is a necessary condition for observing synchronization with a lag time $\tau-\tau_{c}{ }^{4}$ Since $\gamma_{m}, \gamma_{s}$, and $\eta$ cannot take negative values, the maximum value of $\eta$ in Fig. 2 is equal to $\gamma_{m}\left(=20 \mathrm{~ns}^{-1}\right)$.

The dark regions in Figs. 1 and 2 correspond to regions in which there is synchronization. In Fig. 1, the degree of synchronization is very good: The correlation coefficient is larger than 0.99 in the 


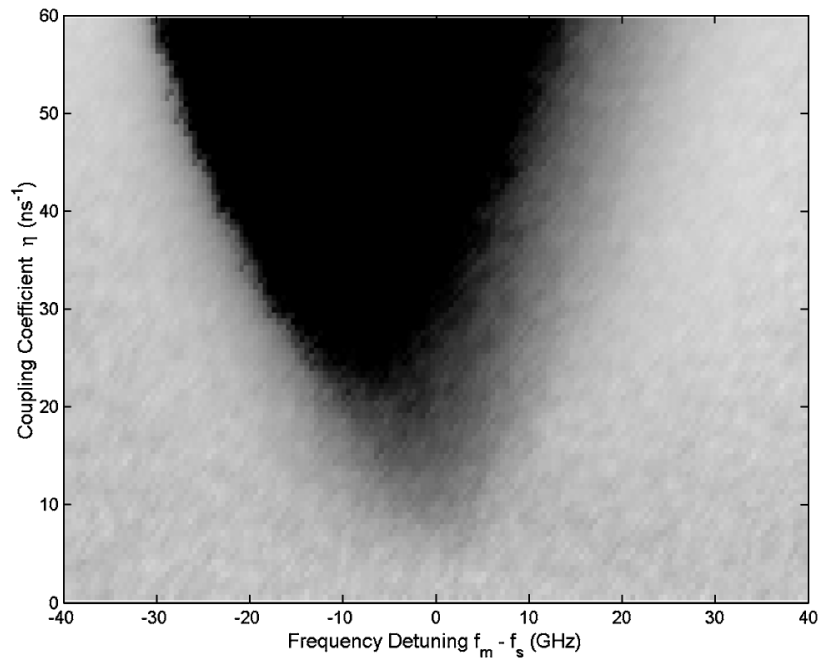

Fig. 1. Correlation coefficients $C$ as a function of the frequency detuning and the optical coupling strength. The parameters are $\gamma_{m}=\gamma_{s}=20 \mathrm{~ns}^{-1}, k=250 \mathrm{~ns}^{-1}, \epsilon=0.017$, $\tau_{n}=2 \mathrm{~ns}, \alpha=5, j=8.25, D=0.02 \mathrm{~ns}^{-1}$, and $\omega_{m} \tau=$ $-2.5 \mathrm{rad}$. In the synchronization region, $C$ is larger than 0.99 .

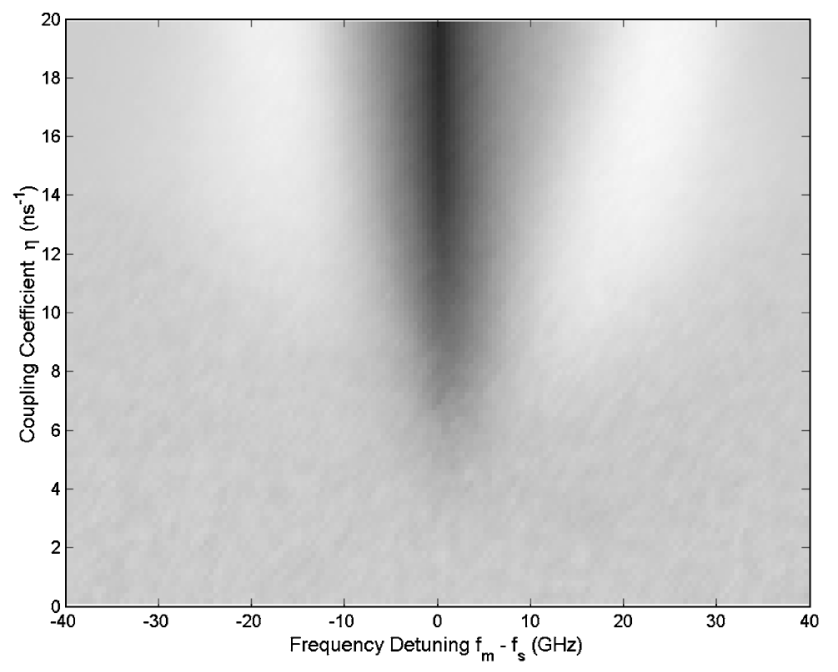

Fig. 2. Same as Fig. 2 but with the feedback of the slave laser $\gamma_{s}=\gamma_{m}-\eta$. To facilitate the comparison, we plot the correlation coefficient on the same gray scale as in Fig. 1. The largest value of $C$ is 0.94 .

synchronization region (the coefficient decreases near the boundaries of the region, abruptly for negative detuning and gradually for positive detuning). In Fig. 2, the degree of synchronization is not as good: The maximum value of the correlation coefficient is 0.94 (notice the difference from the dark gray of the synchronization regions in Figs. 1 and 2). One can see that, for the first type of synchronization to occur, coupling coefficient $\eta$ must be at least larger than $\gamma_{m}\left(=20 \mathrm{~ns}^{-1}\right)$. However, the second type of synchronization occurs for coupling strengths that are at least larger than $\gamma_{m} / 2\left(=10 \mathrm{~ns}^{-1}\right)$.

The sensitivity to frequency detuning is different for each type of synchronization. In Fig. 1, the synchronization region extends over detunings of a few tens of gigahertz, whereas in Fig. 2 the synchronization region extends over detunings of only a few gigahertz. The reason for this difference is twofold. First, the coupling strength can be much larger in the first type of synchronization than in the second (since in the latter case the coupling strength is inherently limited to a maximum value of $\eta=\gamma_{m}$ ). The second reason is the existence, in the first type of synchronization, of a mechanism that compensates for the effect of frequency detuning, which is absent in the second type. In the second type of synchronization, the lagged laser variables (the optical field and the carrier density) are exactly equal. ${ }^{4}$ In the first type of synchronization, the laser variables are related but are not necessarily equal. ${ }^{15}$ The difference between the laser variables varies with the amount of frequency detuning in such a way that synchronization can be preserved. These variations (that compensate for frequency detuning) are not possible in the second regime of synchronization (in which the laser variables are identical and the presence of frequency detuning cannot be compensated for). The fact that these variations are not possible contributes to an explanation of the lessrobust synchronization in the second case.

In Fig. 2, there are two parameter regions in which the correlation coefficient takes a negative value (notice the lighter zones surrounding the dark synchronization zone). The behavior in these regions might be related to the antisynchronization phenomenon that was recently reported in Refs. 12 and 14 . However, the correlation coefficient is at most -0.3 , so the lasers are not antisynchronized.

The second regime of synchronization is more sensitive to noise than the first. In Fig. 1, the correlation coefficient is larger than 0.999 even in the presence of noise. However, in Fig. 2, and without detuning, the correlation coefficient is at most 0.939 , whereas in the absence of noise the intensities would be perfectly synchronized (with a correlation coefficient equal to 1). One can better represent the effect of noise visually by plotting the intensity of the slave laser versus the intensity of the master laser. Figure 3(a) corresponds to $\gamma_{m}=\gamma_{s}$, and $I_{m}$ is shifted in time by $\tau_{l}=-\tau_{c}$; Fig. 3(b), to $\gamma_{m}=\gamma_{s}+\eta$, and $I_{m}$ is shifted in time by $\tau_{l}=\tau-\tau_{c}$. The other parameters are chosen from the synchronization regions of Figs. 1 and 2. The points in Fig. 3(a) are clearly concentrated along a straight line, whereas in Fig. 3(b) they are more scattered (owing to noise-induced bursts of desynchronization). Notice that in Fig. 3(a) the points lie along a line that is slightly tilted with respect to the diagonal, whereas in Fig. 3(b) most of the points are aligned along the diagonal. This difference is due to the fact that, when the lasers have identical parameters, in the first type of synchronization, $I_{s}(t)=a I_{m}\left(t-\tau_{c}\right)$, where $a$ is a constant, ${ }^{15}$ whereas in the second type, $I_{s}(t)=I_{m}\left(t-\tau_{c}+\tau\right){ }^{4}$

We now address the results obtained when a small sinusoidal modulation is applied to the injection current of the master laser: $j_{m}=j_{0}\left[1+h \sin \left(2 \pi f_{\bmod } t\right)\right]$. We are motivated by the experimental results of Fischer et al. ${ }^{10}$ and we chose parameters to match the relaxation oscillation frequency and the external-cavity frequency of the lasers used in the study reported in 

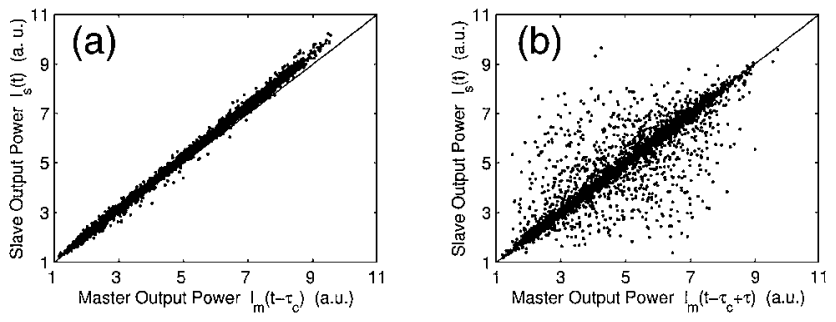

Fig. 3. (a) $I_{m}\left(t-\tau_{c}\right)$ plotted versus $I_{s}(t)$. The parameters are $\gamma_{m}=\gamma_{s}=20 \mathrm{~ns}^{-1}, \eta=50 \mathrm{~ns}^{-1}, \Delta \omega=0$, and $D=$ $0.02 \mathrm{~ns}^{-1}$; all other parameters are as in Fig. 2 . $C=0.99$. (b) $I_{m}\left(t+\tau-\tau_{c}\right)$ plotted versus $I_{s}(t)$. The parameters are $\gamma_{m}=20 \mathrm{~ns}^{-1}, \gamma_{s}=0, \eta=20 \mathrm{~ns}^{-1}, \Delta \omega=0$, and $D=$ $0.02 \mathrm{~ns}^{-1}$; all other parameters are as in Fig. 2. $C=0.94$.
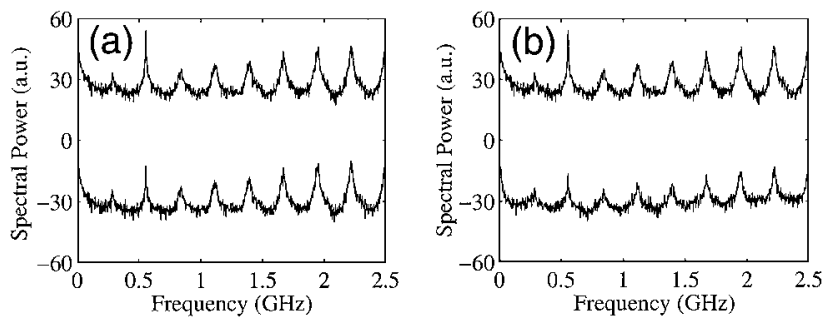

Fig. 4. rf spectra of the lasers when the master injection current is periodically modulated. The top (bottom) trace corresponds to the master (slave) laser. The parameters are $\alpha=3, \tau_{n}=1 \mathrm{~ns}, k=300 \mathrm{~ns}^{-1}, \epsilon=0.01, D=0.01 \mathrm{~ns}^{-1}$, $j_{0}=1.5, h=0.1, \tau=3.6 \mathrm{~ns}, \tau_{c}=1.7 \mathrm{~ns}$, and $\gamma_{m}=50 \mathrm{~ns}^{-1}$. (a) $\gamma_{s}=\gamma_{m}, \eta=3 \gamma_{m}$. $C=0.98$. (b) $\gamma_{s}=0, \eta=\gamma_{m}$. C $=0.80$.

Ref. 10. Figure 4 shows the rf spectra of the lasers for two synchronization regimes, for a modulation frequency that is related to the frequencies appearing on the deterministic dynamics $\left(f_{\bmod }=2 / \tau\right)$. The spectrum of the slave laser is shifted down for clarity. Figure 4(a) corresponds to $\gamma_{m}=\gamma_{s}$; Fig. 4(b), to $\gamma_{m}=\eta$ (as in Ref. 10, we consider the slave laser a solitary laser). Notice that the slave laser reproduces the spectrum of the master laser, with the peak corresponding to the frequency of the modulation attenuated in both types of synchronization. However, in the first type of synchronization the spectrum is reproduced with all its details, whereas in the second type, small differences can be observed.

To summarize, we have compared two types of synchronization of unidirectionally coupled externalcavity semiconductor lasers. In the first type, the lasers are subjected to the same feedback level, and the laser variables are functionally related, with the lag time between them equal to $-\tau_{c}$. In the second type, the lasers are subjected to the same amount of total optical injection, and the laser variables are identical, with a lag time $\tau-\tau_{c}$.

In the first regime of synchronization, a parameter region exists (for large-enough coupling) such that there is good synchronization even when noise and frequency detuning (of several gigahertz) are taken into account. In contrast, the second regime of synchronization is much more sensitive to frequency detuning and noise. These results suggest that the first type of synchronization might be easier to realize in practical schemes, such as cryptography, than the second type.

This research was supported by the InterUniversity Attraction Pole Program (IAP IV/07) of the Belgian government and by the Fonds $\mathrm{Na}-$ tional de la Recherche Scientifique. C. Masoller is partially supported by the Program de Desarrollo de las Ciencias Básicas and the Consejo Superior de Investigaciones Cientificas, Uruguay. A. Locquet's e-mail address is aloquet@georgiatech-metz.fr.

*Present address, GTL-CNRS Telecom, Unité Mixte de Recherche Centre National de la Recherche Scientifique 6603, Georgia Tech Lorraine.

\section{References}

1. C. R. Mirasso, P. Colet, and P. Garcia-Fernandez, IEEE Photon. Technol. Lett. 8, 299 (1996).

2. G. D. VanWiggeren and R. Roy, Science 279, 1200 (1998).

3. J.-P. Goedgebuer, L. Larger, and H. Porte, Phys. Rev. Lett. 80, 2249 (1998).

4. V. Ahlers, U. Parlitz, and W. Lauterborn, Phys. Rev. E 58, 7208 (1998).

5. P. S. Spencer and C. R. Mirasso, IEEE J. Quantum Electron. 35, 803 (1999).

6. J. K. White and J. V. Moloney, Phys. Rev. A 59, 2422 (1999).

7. S. Sivaprakasam and K. A. Shore, Opt. Lett. 24, 1200 (1999).

8. Y. Takiguchi, H. Fujino, and J. Ohtsubo, Opt. Lett. 24, 1570 (1999).

9. H. Fujino and J. Ohtsubo, Opt. Lett. 25, 625 (2000).

10. I. Fischer, Y. Liu, and P. Davies, Phys. Rev. A 62, 011801(R) (2000).

11. C. Masoller, Phys. Rev. Lett. 86, 2782 (2001).

12. I. Wedekind and U. Parlitz, Int. J. Bifurcation Chaos 11, 1141 (2001).

13. Y. Liu, H. F. Chen, J. M. Liu, P. Davis, and T. Aida, Phys. Rev. A 63, 031802(R) (2001).

14. S. Sivaprakasam, I. Pierce, P. Rees, P. S. Spencer, K. A. Shore, and A. Valle, Phys. Rev. A 64, 013805 (2001).

15. A. Locquet, F. Rogister, M. Sciamanna, P. Megret, and M. Blondel, Phys. Rev. E 64, 045203(R) (2001). 